\title{
Gb/s Single-LED OFDM-based VLC Using Violet and UV Gallium Nitride $\mu$ LEDs
}

\author{
Jonathan J.D. McKendry ${ }^{1}$, Dobroslav Tsonev ${ }^{2}$, Ricardo Ferreira ${ }^{1}$, Stefan Videv ${ }^{2}$, Alexander D. Griffiths ${ }^{1}$, \\ Erdan $\mathrm{Gu}^{1}$, Anthony E. Kelly ${ }^{3}$ Harald Haas ${ }^{2}$ and Martin D. Dawson ${ }^{1}$. \\ *Corresponding author: jonathan.mckendry@strath.ac.uk \\ 1) Institute of Photonics, University of Strathclyde, Glasgow G4 0NW, U.K. \\ 2) Institute for Digital Communications, Joint Research Institute for Signal and Image Processing, The University of Edinburgh, Edinburgh \\ EH9 3JL, U.K. \\ 3) School of Engineering, University of Glasgow, Glasgow G12 8QQ, U.K.
}

\begin{abstract}
We present data transmission achieved using orthogonal frequency division multiplexing (OFDM) together with violet $(405 \mathrm{~nm})$ and ultraviolet $(370 \mathrm{~nm})$ micro-sized light-emitting diodes ( $\mu$ LEDs), at data rates of 3.32 and 1.31 $\mathrm{Gb} / \mathrm{s}$, respectively.
\end{abstract}

I. INTRODUCTION

Visible light communications that utilize white light-emitting diodes (LEDs) for both illumination and communication (' $\mathrm{Li}-\mathrm{Fi}$ ') has emerged as a timely solution for addressing the challenges posed by the exponential growth in wireless data traffic. Li-Fi allows lighting infrastructure to provide access to $100 \mathrm{~s}$ of THz of licencefree bandwidth for wireless communications, in a configuration that does not interfere with existing RF technology, thereby addressing the impending "spectrum crisis".

The most common format of white LED consists of a blue-emitting LED integrated with a yellow-emitting phosphor. The blue emission from the LED is partially converted to yellow, giving an overall white emission. One shortcoming of this implementation is that these LEDs have a poor color-rendering index (CRI). In addition, the yellow phosphor has a long $(\mu s)$ excited-state lifetime, so it is not well suited for high-speed VLC.

An alternative approach would be to combine short-wavelength LEDs with alternative color-converters such lightemitting polymers (LEPs) or inorganic semiconductor membranes. We have previously demonstrated blue $\mu$ LEDs combined with LEPs [1] and inorganic semiconductor membranes [2] for VLC. Here we report $\mu$ LEDs emitting at shorter wavelengths, ultraviolet (UV, approximate peak wavelength $370 \mathrm{~nm})$ and violet $(405 \mathrm{~nm})$. These wavelengths are viable alternatives for generating white light via color-converters, whilst also enabling techniques such as self-aligned patterning of LEPs [3]. Using orthogonal frequency division multiplexing (OFDM) data rates of 3.32 and $1.31 \mathrm{~Gb} / \mathrm{s}$, are achieved using violet and UV $\mu \mathrm{LEDs}$, respectively. This work provides a baseline for future work utilising white LEDs based on UV/violet LEDs and color-converting materials.

\section{II. $\quad \mu$ LED DESIGN AND PERFORMANCE}

Conventional LED die typically consist of a single active area of the order of $1 \mathrm{~mm}^{2}$. In contrast, the Gallium Nitride (GaN)-based $\mu$ LED devices used in this work are comprised of arrays of individually-addressable pixels, each with dimensions $<100 \mu \mathrm{m}$. We have previously shown that, due to their small size and ability to be driven at much higher current densities than conventional LEDs, $\mu$ LEDs offer significantly higher modulation bandwidths, in excess of $400 \mathrm{MHz}$ in some cases [4]. Furthermore, the array format of the $\mu$ LEDs also offers the possibility to use multiple pixels within the array to transmit multiple data streams simultaneously.

For these reasons $\mu$ LEDs were chosen as the optical transmitters as opposed to a more conventional LED. The UV and violet $\mu$ LED arrays had peak wavelengths of approximately 370 and $405 \mathrm{~nm}$, respectively. Single pixels from each array were used, with diameters of 60 and $40 \mu \mathrm{m}$ for the UV and violet devices, respectively. The light output power-current-voltage (L-I-V) characteristics of the devices are shown in Figure 1 (a). Maximum output powers were measured to be $1.5 \mathrm{~mW}$ for the $\mathrm{UV} \mu \mathrm{LED}$, and $2.5 \mathrm{~mW}$ for violet.
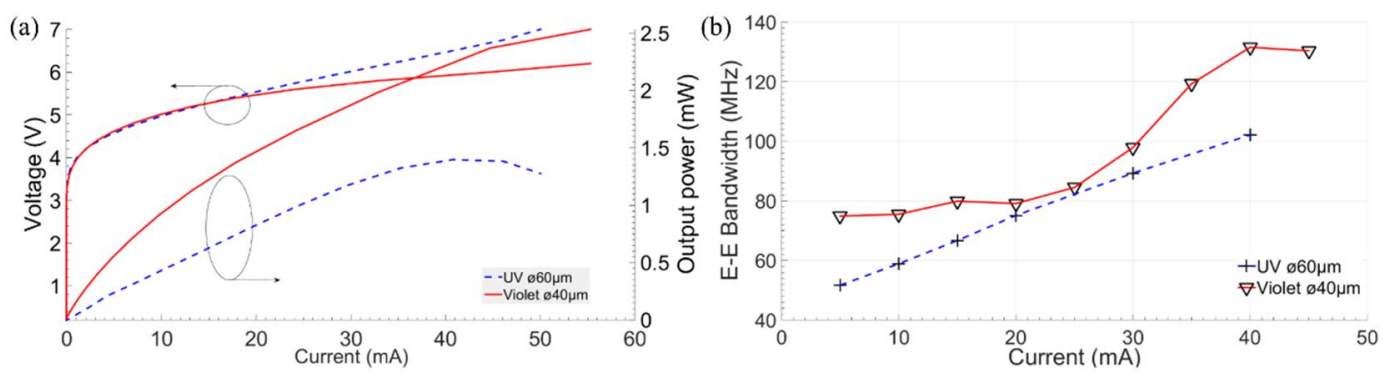

Figure 1 - (a) output power and voltage vs. current and (b) electrical -3dB bandwidth versus current for UV and violet $\mu$ LEDs.

The small-signal modulation bandwidths of the $\mu$ LEDs were measured using a similar method as described in [4]. As shown in Figure 1(b), the modulation bandwidths of the devices increase with increasing carrier density, as 
discussed in [4]. Maximum electrical-to-electrical $-3 \mathrm{~dB}$ bandwidths of 100 and 130 were found for the UV and violet devices, respectively.

\section{DATA TRANSMISSION USING OFDM}

With the exception of different optics and photoreceiver the experimental setup used for transmission of data using the $\mu$ LEDs is otherwise the same as in our previous publication [5], and for brevity will not be described in full detail here. Using MATLAB ${ }^{\circledR}$ a pseudo-random bit stream is encoded into $M$-ary quadrature amplitude modulation ( $M$-QAM) symbols, and converted to a discrete time-domain signal by an inverse fast Fourier transform (IFFT). This signal is subsequently used to modulate the $\mu$ LED output via an arbitrary function generator, amplifier and bias-tee. The optical emission from the $\mu$ LEDs were then collected and imaged onto a fast photoreceiver (Femto HAS-X-S-1G4-Si, $1.4 \mathrm{GHz}$ bandwidth), amplified and the received waveforms recorded by a digital oscilloscope. Finally, the received waveforms were processed in MATLAB ${ }^{\circledR}$ to obtain the received equalized $M$-QAM symbols.

For the $60 \mu \mathrm{m}$ diameter UV $\mu \mathrm{LED}$, optimum bias conditions were found to consist of a $2 \mathrm{~V}$ peak-to-peak $\left(\mathrm{V}_{\mathrm{pk}-\mathrm{pk}}\right)$ signal with an average DC current of $15 \mathrm{~mA}$. Similarly for the $40 \mu \mathrm{m}$ diameter violet $\mu \mathrm{LED}$, an optimum $3 \mathrm{~V}_{\mathrm{pk}-\mathrm{pk}}$ and DC current $25 \mathrm{~mA}$ was used.

An adaptive algorithm was used to estimate the optimal bit and energy loading of the sub-carriers by attempting to ensure a constant (signal-to-noise ratio) SNR for all subcarriers of a particular QAM constellation size. Using this method, for the UV device a data rate of $1.41 \mathrm{~Gb} / \mathrm{s}$ with a bit-error rate of $2.46 \times 10^{-3}$ was achieved, and 3.57 $\mathrm{Gb} / \mathrm{s}$ with a BER of $2.1 \times 10^{-3}$ for the violet $\mu \mathrm{LED}$. Taking into account a $7 \%$ overhead for forward-error correction (FEC) the overall data rates are 1.31 and $3.32 \mathrm{~Gb} / \mathrm{s}$ for the UV and violet $\mu L E D s$, respectively. These represent the fastest single-link LED-based communication system demonstrations operating at UV and violet wavelengths.
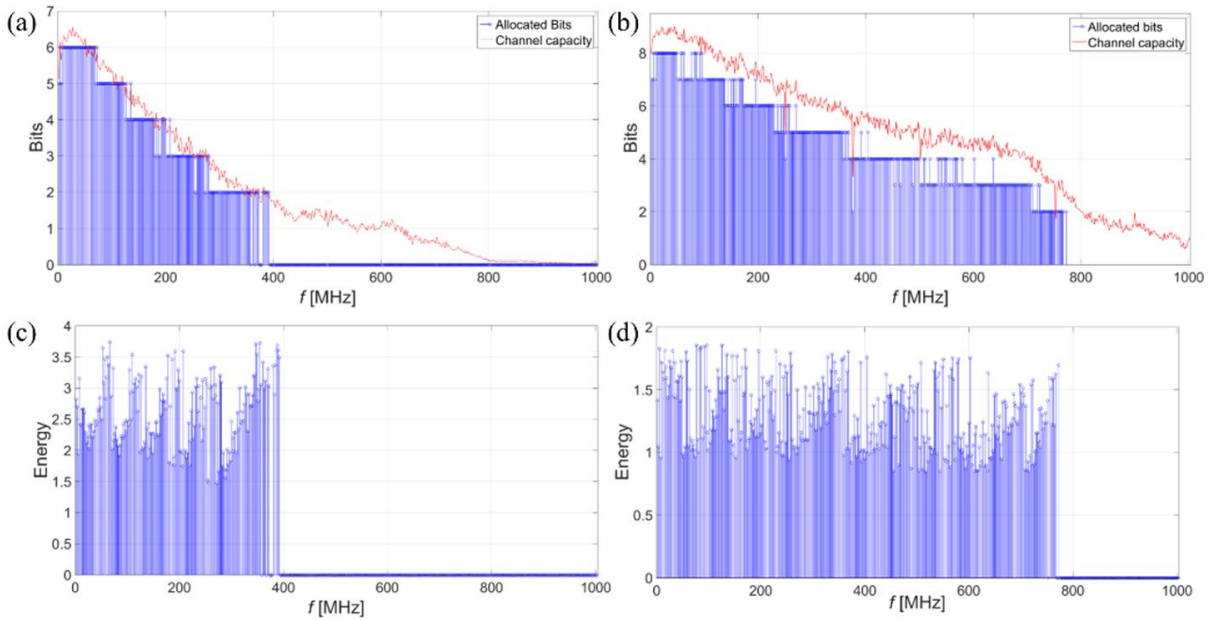

Figure 2 -allocated bits $(a, b)$ and energy $(c, d)$ for $U V$ and violet $\mu L E D$ s, respectively. The energy is normalized to the average energy in the OFDM frame.

\section{SUMMARY}

Wireless VLC links using single GaN $\mu$ LEDs emitting at 370 and $405 \mathrm{~nm}$ have been demonstrated transmitting data at rates of 1.31 and $3.32 \mathrm{~Gb} / \mathrm{s}$, respectively. These results represent the fastest transmission rates demonstrated using a single LED at these wavelengths, and provide baseline results for future work converting these wavelengths to white light.

\section{REFERENCES}

[1] H. Chun, P. Manousiadis, S. Rajbhandari, D. A. Vithanage, G. Faulkner, D. Tsonev, J. J. D. McKendry, S. Videv, E. Xie, E. Gu, M. D. Dawson, H. Haas, G. A. Turnbull, I. D. W. Samuel, and D. C. O'Brien, "Visible Light Communication Using a Blue GaN $\mu \mathrm{LED}$ and Fluorescent Polymer Color Converter," IEEE Photonics Technol. Lett., vol. 26, no. 20, pp. 2035-2038, Oct. 2014.

[2] J. M. M. Santos, B. E. Jones, P. J. Schlosser, S. Watson, J. Herrnsdorf, B. Guilhabert, J. J. D. McKendry, J. De Jesus, T. A. Garcia, M. C. Tamargo, A. E. Kelly, J. E. Hastie, N. Laurand, and M. D. Dawson, "Hybrid GaN LED with capillary-bonded II - VI MQW color-converting membrane for visible light communications," Semicond. Sci. Technol., vol. 30, no. 3, p. 035012 , 2015.

[3] B. Guilhabert, D. Elfström, A. J. C. Kuehne, D. Massoubre, H. X. Zhang, S. R. Jin, A. R. Mackintosh, E. Gu, R. A.
Pethrick, and M. D. Dawson, "Integration by self-aligned writing of nanocrystal/epoxy composites on InGaN micro-pixelated lightemitting diodes," Opt. Express, vol. 16, no. 23, pp. 18933-18941, 2008 .

[4] J. J. D. McKendry, D. Massoubre, S. Zhang, B. R. Rae, R. P. Green, E. Gu, R. K. Henderson, A. E. Kelly, and M. D. Dawson, "Visible-Light Communications Using a CMOS-Controlled MicroLight- Emitting-Diode Array," J. Light. Technol., vol. 30, no. 1, pp. 61-67, Jan. 2012.

[5] D. Tsonev, H. Chun, S. Rajbhandari, J. J. D. McKendry, S. Videv, E. Gu, M. Haji, S. Watson, A. E. Kelly, G. Faulkner, M. D. Dawson, H. Haas, and D. O. Brien, "A 3-Gb/s Single-LED OFDMBased Wireless VLC Link Using a Gallium Nitride $\mu$ LED," IEEE Photonics Technol. Lett., vol. 26, no. 7, pp. 637-640, 2014. 